\title{
Static and Dynamic Chain Structures in the Mean-Field Theory
}

\author{
T. Ichikawa ${ }^{1}$, N. Itagaki ${ }^{1}$, N. Loebl ${ }^{2}$, J. A. Maruhn ${ }^{2,1 a}$, V. E. Oberacker ${ }^{3}$, S. Ohkubo $^{4}$, B. Schuetrumpf ${ }^{2}$, and A. S. Umar ${ }^{3}$ \\ 1 Yukawa Institute for Theoretical Physics, Kyoto University, Kyoto 606-8502, Japan \\ 2 Institut fuer Theoretische Physik, Goethe-Universitaet, Frankfurt am Main, Germany \\ 3 Department of Physics and Astronomy, Vanderbilt University, Nashville, Tennessee 37235, USA \\ 4 Department of Applied Science and Environment, University of Kochi, Kochi 780-8515, Japan
}

\begin{abstract}
We give a brief overview of recent work examining the presence of $\alpha$-clusters in light nuclei within the Skyrme-force Hartree-Fock model. Of special signif cance are investigations into $\alpha$-chain structures in carbon isotopes and ${ }^{16} \mathrm{O}$. Their stability and possible role in fusion reactions are examined in static and time-dependent Hartree-Fock calculations. We f nd a new type of shape transition in collisions and a centrifugal stabilization of the $4 \alpha$ chain state in a limited range of angular momenta. No stabilization is found for the $3 \alpha$ chain.
\end{abstract}

\section{Introduction}

In light nuclei, cluster structures and large deformation may be present even in the mean-f eld model [1]. For example, ${ }^{8} \mathrm{Be}$ has an $\alpha$ - $\alpha$ structure of "superdeformed" nature, and the degree of deformation is closely related to the orbits of valence neutrons in neutron-rich Be isotopes [2, 3]. Also there have been many discussions on the nature of the $0_{2}^{+}$state of ${ }^{12} \mathrm{C}$ with well-developed $3 \alpha$ structure, possibly a linear chain [4-7]. Another example is the $4 \alpha$ linear chain band starting around the $4 \alpha$ threshold energy region in ${ }^{16} \mathrm{O}$ suggested by Chevallier et al. [8] through the data analysis of the ${ }^{12} \mathrm{C}+\alpha \rightarrow{ }^{8} \mathrm{Be}+{ }^{8} \mathrm{Be}$ reaction. This is supported by theoretical work[9]; they analyzed the decay widths and discussed that the observed states are possibly characterized by well-developed $4 \alpha$ structure.

Concerning the stability of the linear chain states, in conventional models, it has been usually studied through the analyses of small vibration around the equilibrium conf guration [10]. However, the bending motion is the most important path for the transition to the low-lying states [11]. Therefore, it is necessary to calculate the stability in a wide wave function space, which covers not only the linear chain conf gurations but also the lower excited states. Utilizing a rather large model space, we have analyzed in neutron-rich $\mathrm{C}$ isotopes that adding valence neutrons increases the stability of the linear chain states with respect to the bending motion $[12,13]$. The basic instability still remains, though.

Even if they are fundamentally unstable, the chain structures could still appear as intermediary conf gurations in a collision involving ${ }^{8} \mathrm{Be}$ and/or $\alpha$-particles, and one of the aims of this paper is to explore this possibility using time-dependent Hartree-Fock (TDHF) methods. It is generally acknowledged that the TDHF theory provides a

a Invited Speaker useful foundation for a fully microscopic many-body theory of low-energy heavy-ion reactions [14]. Earlier TDHF calculations of collisions between light nuclei involving cluster-like conf gurations have been made for the study of nuclear molecular resonances [15]. However, due to the lack of computational resources these calculations suffered from numerical imprecision as well as unphysical symmetry assumptions, such as collisions being restricted to axial symmetry. Current TDHF calculations are performed with high numerical precision and with no symmetry assumptions as well as using modern Skyrme forces. Recently, we have shown that when TDHF is combined with the densityconstraint method [16] dynamical potentials and ion-ion interaction barriers can be accurately reproduced [17-19]. Thus an alternative approach would be to investigate the formation and stability of the linear chain conf guration using the fully microscopic and dynamical TDHF theory.

An important mechanism to stabilize the linear chain state could be the rotation of the system; a large moment of inertia such as in the linear-chain conf guration is favored if large angular momentum is given to the nucleus. If the angular momentum is too high, however, it leads to the $f$ ssion of the nucleus, and there must exist a region of angular momentum where the linear chain conf guration is stabilized. We focus on this mechanism and discuss the angular momentum of the linear-chain conf guration based on cranked Hartree-Fock.

\section{The $3 \alpha$ chain state in nuclear collisions}

In this work we study TDHF collisions which reproduce the linear chain conf guration and subsequently decay to lower-energy conf gurations of the system. To our knowledge such mode changes have never been observed in TDHF calculations previously and appear to simulate the 
suggested astrophysical mechanism for the formation of ${ }^{12} \mathrm{C}$ nuclei.

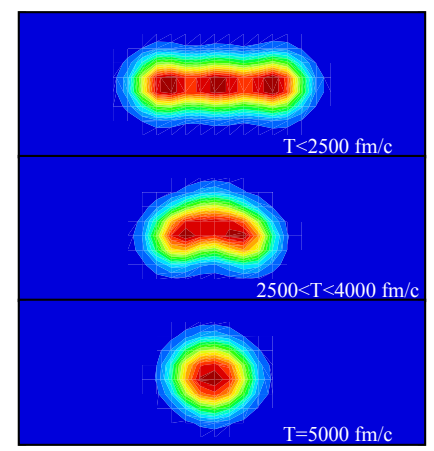

Fig. 1. Selected density prof les from TDHF time-evolution of the ${ }^{4} \mathrm{He}+{ }^{8} \mathrm{Be}$ head-on collision for initial Be orientation angle $\beta=0^{\circ}$ using the SLy4 interaction. The initial energy is $E_{\text {c.m. }}=2 \mathrm{MeV}$.

In our numerical calculations we have chosen a Cartesian box which is $40 \mathrm{fm}$ along the collision axis and $24 \mathrm{fm}$ in the other two directions. Calculations are done in 3-D geometry and using the full Skyrme force (SLy4) [20] as described in Ref. [21]. Using different modern parametrizations of the Skyrme force all show the same phenomena. We have chosen to study two different collisions leading to the linear chain conf guration, frst the ${ }^{4} \mathrm{He}+{ }^{8} \mathrm{Be}$ system and then the triple collision of three ${ }^{4} \mathrm{He}$ nuclei, which may be astrophysically much less probable. The Hartree-Fock (HF) state for the ${ }^{8} \mathrm{Be}$ nucleus is axially symmetric. In Fig. 1 we show three snapshots from the long time evolution of the ${ }^{4} \mathrm{He}+{ }^{8} \mathrm{Be}$ collision at zero impact parameter and $E_{\mathrm{c} . \mathrm{m} .}=2 \mathrm{MeV}$. The top panel of Fig. 1 shows the linear chain conf guration about which the system oscillates for times less than $2,500 \mathrm{fm} / c$. In particular, it is remarkable that the moving clusters do not equilibrate while moving inside the linear chain state but retain their $2 p-2 n$ character, where one observes a complex quasiperiodic motion with little damping up to this time. Around $2500 \mathrm{fm} / c$ the system starts to bend and acquires a somewhat triangular shape as shown in the middle panel of Fig. 1. The system still retains its cluster character with the center cluster moving off the reaction plane cut shown in the f gure, but can be clearly observed in volumetric threedimensional movies of the collision process. The bending motion, where the center cluster oscillates somewhat perpendicular to the left and right clusters continues for approximately $1000 \mathrm{fm} / c$, with very little damping. Finally, at even longer times the system relaxes into a relatively more compact shape (bottom panel of Fig. 1.). Such mode changes, where the dynamical energy in the longitudinal direction is converted to a transverse mode, while the system retains its cluster structure have never been seen in previous TDHF calculations albeit this would not have been possible in calculations imposing axial symmetry. Even in three-dimensional calculations, for an exactly central collision, the axial symmetry would be preserved under ideal theoretical and numerical conditions. Therefore the mean- ing of head-on collision $(b=0)$ should be interpreted to have a small dispersion around this value, which facilitates the mode change even for exactly central collisions.

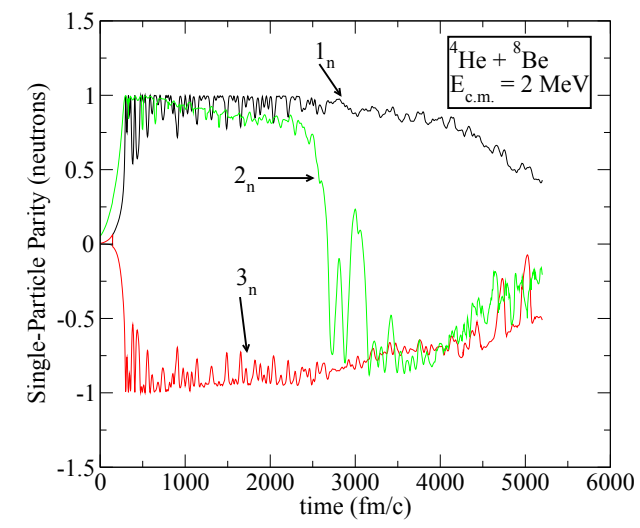

Fig. 2. Single-particle parities of the neutron states during the collision of the ${ }^{4} \mathrm{He}+{ }^{8} \mathrm{Be}$ system as a function of time at $E_{\mathrm{c} . \mathrm{m} .}=$ $2 \mathrm{MeV}$.

We were also successful, for the frst time, in creating a static Hartree-Fock linear chain state orthogonal to the ground state in a fully three-dimensional geometry. This was achieved by initializing one of the single-particle states to be in the $s-d$ shell with positive parity rather than in the $p$ state with negative parity. This results in a linear chain state similar to the one shown in the top panel of Fig. 1. The fact that this state has two positive parity and one negative parity state proves that it is exactly orthogonal to the ${ }^{12} \mathrm{C}$ ground state which has one positive parity and two negative parity states. A similar dependence on parity was also studied in cluster model calculations [22]. In order to relate this observation to the dynamical mode changes discussed above we have used the density constraint method to calculate the potential energy and the single-particle parities during the TDHF time-evolution. In Fig. 2 we show the neutron single-particle parities as a function of collision time. What is striking is that the combined system initially has the same parity signature as the static linear chain state but at the time of bending one of the positive parity states starts to decay towards negative parity and this decay continues as the system becomes closer to the parity signatures of the ground state. Oscillations in the numerically calculated parities stem from the fact that these are done during the dynamical evolution of the system. The proton single-particle parities are almost exactly the same as for the neutrons as anticipated.

In order to relate the observed mode changes more closely to the intrinsic energy of the system we have also calculated the potential energy of the system as a function of the ion-ion separation distance $R$ [17]. For the calculation of $R$ we have used the hybrid method described in [19], which relates $R$ to the quadrupole moment of the system thus making it possible to have a consistent def nition of $R$ for large overlaps. The calculated potential energy curves are shown in Fig. 3 as a function of three alignments of the ${ }^{8} \mathrm{Be}$ nucleus with respect to the collision axis labeled 


\section{FUSION11}

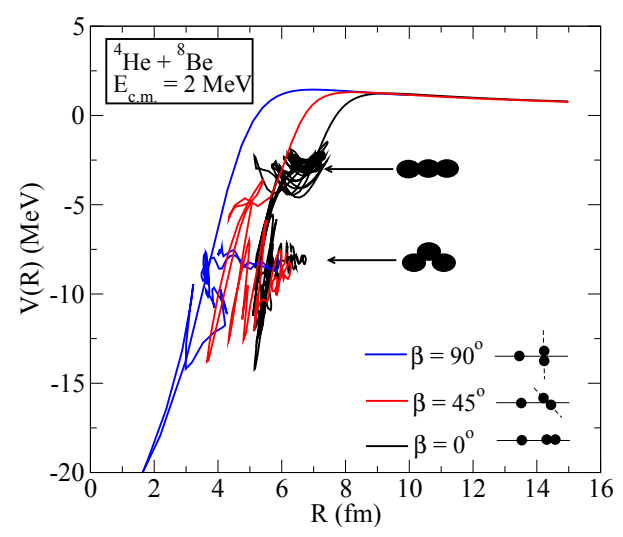

Fig. 3. Potential energy curves for the collision of the ${ }^{4} \mathrm{He}+{ }^{8} \mathrm{Be}$ system as a function of $R$ for three initial alignments of the $\mathrm{Be}$ nucleus and at $E_{\mathrm{c.m} .}=2 \mathrm{MeV}$.

as angle $\beta$, and for the entire duration of the collision process. Since for the real system the angular momentum of ${ }^{8} \mathrm{Be}$ is projected all possible alignments of the ${ }^{8} \mathrm{Be}$ nucleus needs to be considered. For all of the alignments the combined system climbs up a shallow potential barrier height of approximately $1.24-1.44 \mathrm{MeV}$, the lowest barrier being that of the $\beta=0^{\circ}$ potential, thus making this alignment most probable under threshold conditions. For the potential energy curve showing the head-on collision $\left(\beta=0^{\circ}\right.$, black curve) we observe that the system initially relaxes to a relatively shallow metastable minimum and oscillates about this minimum until approximately $T=2500 \mathrm{fm} / \mathrm{c}$ at which points it slips down the curve towards the second conf guration as indicated by three alphas in a triangular conf guration. After spending some time in this conf guration the system further slips down to even lower energy and more compact conf guration. The potential energy curves corresponding to ${ }^{8} \mathrm{Be}$ initial alignment angles of $\beta=45^{\circ}$ and $\beta=90^{\circ}$ (red and blue curves, respectively) undergo a different behavior, bypassing the linear chain minimum but directly going to the triangular and subsequently to the compact conf guration, the perpendicular energy collision attaining the most compact and lowest energy conf guration. It is interesting to note that all of the potential energy curves spend some time in the triangular conf guration.

As an alternate collision leading to the same conf guration we have also studied the collision of three ${ }^{4} \mathrm{He}$ nuclei, one at rest at the origin of the collision axis and the other two on each side boosted towards the center with $1 \mathrm{MeV}$ energy. In Fig. 4 we contrast the time dependence of the potential energies for the two different collisions. We observe that the three ${ }^{4} \mathrm{He}$ collision process spends considerably longer time (about $6000 \mathrm{fm} / \mathrm{c}$ ) undergoing quasiperiodic oscillations with very little damping in the linear chain conf guration before switching to bending and compact modes.

In order to gauge the stability of the linear chain conf guration we have made systematic studies as a function of impact parameter and center-of-mass energy, as well as a study using heavier Be isotopes to determine the dependence on neutron number. In Fig. 5 we show the depen-

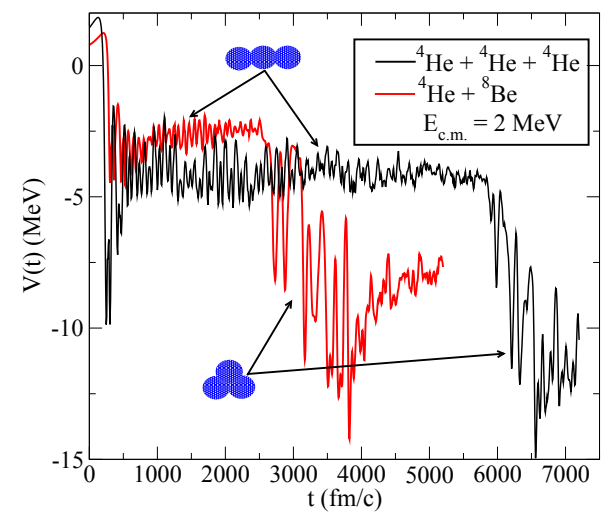

Fig. 4. Time development of the potential energy for the headon collision of the ${ }^{4} \mathrm{He}+{ }^{8} \mathrm{Be}$ and the ${ }^{4} \mathrm{He}+{ }^{4} \mathrm{He}+{ }^{4} \mathrm{He}$ systems for $E_{\text {c.m. }}=2 \mathrm{MeV}$.

dence of the linear chain survival time on the impact parameter for the ${ }^{4} \mathrm{He}+{ }^{8} \mathrm{Be}$ system at $E_{\text {c.m. }}=2 \mathrm{MeV}$ and $\beta=0^{\circ}$. We observe that as the impact parameter increases the survival time rapidly decreases. This decrease naturally happens slower (faster) for lower (higher) energies. We have also done a similar study for the time spent in the linear chain conf guration as a function of the center-of-mass energy for the ${ }^{4} \mathrm{He}+{ }^{8} \mathrm{Be}$ system for $\beta=0^{\circ}$ alignment. We decreased the energy in steps of $0.1 \mathrm{MeV}$ to $\mathrm{f}$ nd the lowest energy for which we form the linear chain conf guration (at lower energies the nuclei rebound due to Coulomb repulsion). At this energy of $1.3 \mathrm{MeV}$ the lifetime of the linear chain conf guration increases to about $2875 \mathrm{fm} / c$. As the energy is increased the lifetime decreases gradually. In order to study the dependence of the linear chain state on the neutron number of the Be isotopes we have repeated all of the above calculations using a ${ }^{9} \mathrm{Be}$ nucleus instead. The calculations were done by using all the time-odd terms in the Skyrme interaction appropriate for an odd-A nucleus. While we do fnd an analogous behavior in this study, the lifetime of the linear chain state is approximately $30 \%$ less than that of the corresponding ${ }^{8} \mathrm{Be}$ system.

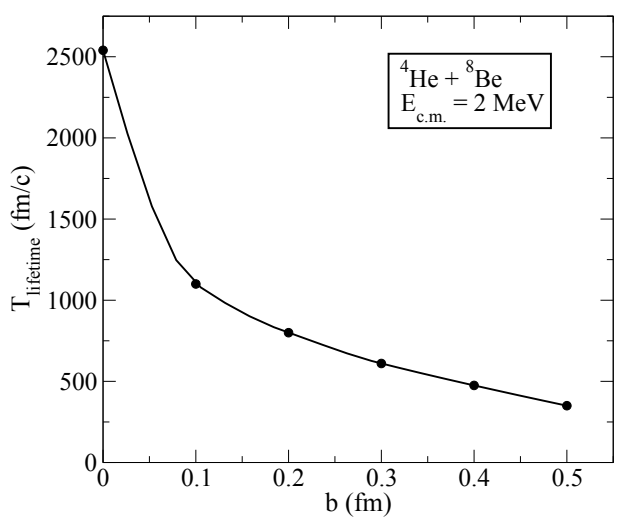

Fig. 5. Time spent in the linear chain conf guration as a function of the impact parameter $b$ for the ${ }^{4} \mathrm{He}+{ }^{8} \mathrm{Be}$ system at $E_{\mathrm{c} . \mathrm{m} .}=$ $2 \mathrm{MeV}$ and $\beta=0^{\circ}$ alignment. 


\section{Cranked $4 \alpha$ chain states}

To discuss the $4 \alpha$ linear chain state in the rotational frame, we perform cranked HF calculations. We self-consistently calculate the cranked HF equation, given by $\delta\langle H-\omega J\rangle=$ 0 , where $H$ is the total Hamiltonian, $\omega$ is the rotational frequency, and $J$ is the angular momentum around the $y$ axis. We also perform TDHF calculations in order to discuss the $4 \alpha$ linear chain state in a collision situation.

We represent the single-particle wave functions on a Cartesian grid with a grid spacing of $0.8 \mathrm{fm}$. The grid size is typically $24^{3}$ for ground states and $32 \times 24^{2}$ for superdeformed states. This accuracy was seen to be sufficient to provide converged conf gurations. The numerical procedure is the damped-gradient iteration method [23], and all derivatives are calculated using the Fourier transform method.

We take three different Skyrme forces: Sly6 as a recent $\mathrm{ft}$ which includes information on isotopic trends and neutron matter [24], and SkI3 as well as SkI4 as recent $\mathrm{fts}$ which map the relativistic isovector structure of the spinorbit force [25]. SkI3 contains a f xed isovector part analogous to the relativistic mean-f eld model, whereas SkI4 is adjusted allowing free variation of the isovector spin-orbit term. Thus all forces differ somewhat in their actual shell structure. Besides the effective mass, the bulk parameters (equilibrium energy and density, incompressibility, symmetry energy) are comparable.

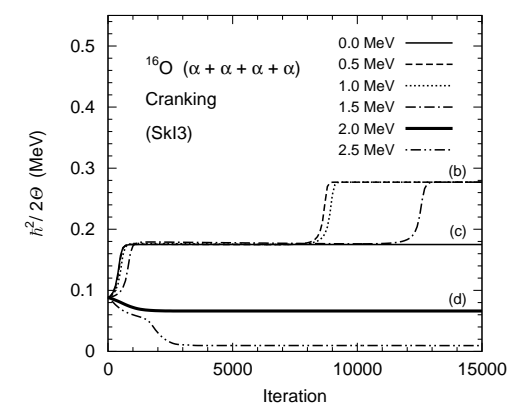

Fig. 7. Coefficient of the rotational energy, $\hbar^{2} / 2 \Theta$, calculated using the cranking method versus the HF iterations with various rotational frequencies $\omega$. The symbols (b), (c), and (d) correspond to the density distribution given in Fig. 6.

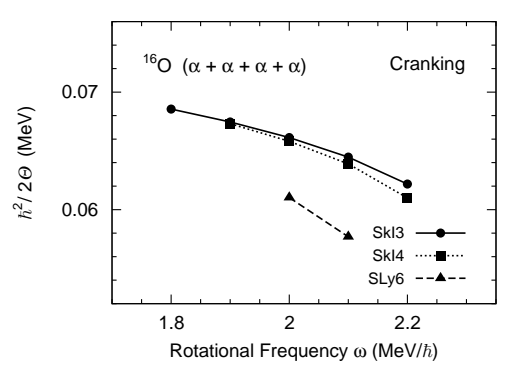

Fig. 8. Coefficient of the rotational energy, $\hbar^{2} / 2 \Theta$, calculated using the cranking method versus the rotational frequency $\omega$. The lines correspond to the different Skyrme forces as indicated.

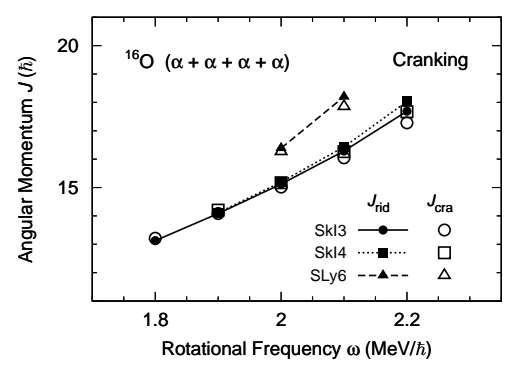

Fig. 9. Angular momentum calculated using the cranking method versus the rotational frequency. The lines with solid symbols denote the calculated results for the rigid-body moment of inertia, while the open symbols denote the results for the cranking method; both for the Skyrme forces as indicated.

We here discuss the stability of the $4 \alpha$ linear chain conf guration in the rotating frame for ${ }^{16} \mathrm{O}$. To this end, we perform the cranked HF calculations with various rotational frequencies, $\omega$. For the initial wave function, we use the twisted $4 \alpha$ conf guration, as shown in Fig. 6 (a). Note that this initial state violates axial symmetry, which facilitates the transition of the initial state to low-lying states including the ground state during the convergence process (this was demonstrated for the carbon chain states in $[12,13])$. We calculate the rigid-body moment of inertia, $\Theta$, using the total nucleon density at each iteration step. We here only consider rotation around the $y$ axis.

We frst investigate the convergence behavior of the HF iterations. To check this, we calculate the coefficient of the rotational energy, given by $\hbar^{2} / 2 \Theta$, at each iteration step. Figure 7 shows the calculated results with various rotational frequencies versus the iterations in the case of the SkI3 interaction. The initial state with the twisted linear chain conf guration is not the true ground state of the HF model space and the solution changes into the true ground state after some large number of iterations; however the situation depends on the value of the rotational frequency $\omega$. In the fure, we see that the rotational frequencies $\omega=0.5,1.0$, and $1.5 \mathrm{MeV} / \hbar$ (the dashed, dotted, and dot-dashed lines, respectively) lead to the true ground state. The corresponding density distribution at the iteration step of 15000 is plotted in Fig. 6 (b). The frequency $\omega=0.0 \mathrm{MeV} / \hbar$ (the solid line) leads to the quasi-stable one (see Fig. 6 (c)). At around $\omega=2.0 \mathrm{MeV} / \hbar$, we obtain the state (the thin solid line) with the $4 \alpha$ linear chain conf guration, as shown in Fig. 6 (d), whereas f ssion occurs above those rotational frequencies (the dot-dot-dashed line).

We next estimate the range of the rotational frequencies where the $4 \alpha$ linear-chain conf guration can be stabilized. Figure 8 shows the coefficient of the rotational energy, $\hbar^{2} / 2 \Theta$, versus the rotational frequency $\omega$ with various Skyrme interactions. We fnd stable states for the $4 \alpha$ linear chain conf guration. For the SkI3 interaction, we obtain the lower and upper bounds of the rotational frequencies as 1.8 and $2.2 \mathrm{MeV} / \hbar$, between these the $4 \alpha$ linear chain conf guration is stabilized. The values are 1.9 and $2.2 \mathrm{MeV} / \hbar$ for the SkI4 interaction and 2.0 and $2.1 \mathrm{MeV} / \hbar$ for the SLy6 interaction, respectively. In these frequency 


\section{FUSION11}

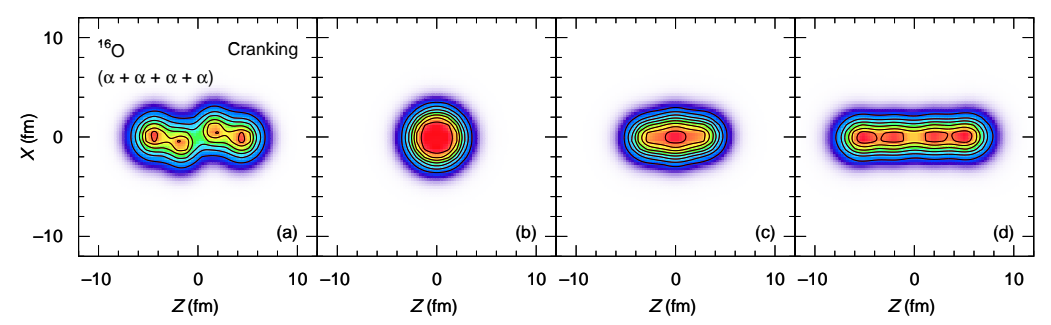

Fig. 6. Total nucleon density distribution calculated using the cranking method for (a) the initial wave function, (b) the ground state, (c) the quasi-stable state, and (d) the $4 \alpha$ linear chain state. The isolines correspond to multiples of $0.02 \mathrm{fm} / \mathrm{c}$. We normalize the color to the density distribution at the maximum of each plot.

regions where the linear chain conf guration is stabilized, we can def ne the rigid-body moments of inertia, which is calculated as $0.065 \mathrm{MeV}$ for the SkI3 and SkI4 interactions and $0.06 \mathrm{MeV}$ for the SLy6 interaction. There values are in very good agreement with the conventional cluster model calculations[26].

We also estimate the corresponding angular momentum where the $4 \alpha$ linear chain conf guration is stabilized. We calculate the angular momentum using the obtained rigid-body moment of inertia and compare those with that calculated by the cranking method. The angular momentum with the rigid-body moment of inertia, $J_{\text {rid }}$, is calculated as $J_{\text {rid }}=\Theta \omega$. The angular moment calculated using the cranking method, $J_{\text {cra }}$, is given by $J_{\text {cra }}=\langle J\rangle$, where $\langle J\rangle$ is the expectation value of the angular momentum in the cranking equation. Figure 9 shows the angular momentum thus obtained versus the rotational frequency. In the f gure, we see that the calculated angular momentum using the rigid-body moment of inertia agrees well with that of the cranking method, indicating that the rigid-body approximation is reasonable for the $4 \alpha$ linear chain states. We fnd that the lower and upper bounds of the angular momentum where the $4 \alpha$ linear chain conf guration is stabilized are about 13 and $18 \hbar$ for the SkI3 interaction, 14 and $18 \hbar$ for the SkI4 interaction, and 16 and $18 \hbar$ for the SLy6 interaction, respectively. With such high angular momenta, very exotic conf guration of $4 \alpha$ linear chain can be stabilized, however, $\mathrm{f}$ ssion occurs beyond this angular momentum region.

We analyzed such stability of linear chain conf gurations of $\alpha$ clusters for three- $\alpha$ in ${ }^{12} \mathrm{C}$ and three- $\alpha$ with valence neutrons in ${ }^{20} \mathrm{C}$. However it is rather difficult to obtain stable regions of rotational frequency and angular momentum in these cases, and stability shown in ${ }^{16} \mathrm{O}$ is considered to be a unique character of the $4 \alpha$ conf guration.

\subsection{Four- $\alpha$ linear chain in collisions of two ${ }^{8} \mathrm{Be}$ with TDHF}

To check the importance of this chain conf guration as an intermediate state in a collision situation, we also performed time-dependent Hartree-Fock calculations for the ${ }^{8} \mathrm{Be}+{ }^{8} \mathrm{Be}$ reaction in a conf guration where the axes of the collision partners are aligned. Fig. 10 shows a number of typical snapshots of the collision for an impact parameter of $6 \mathrm{fm}$ and a relative energy of $2 \mathrm{MeV}$. The $\mathrm{f}$ rst picture

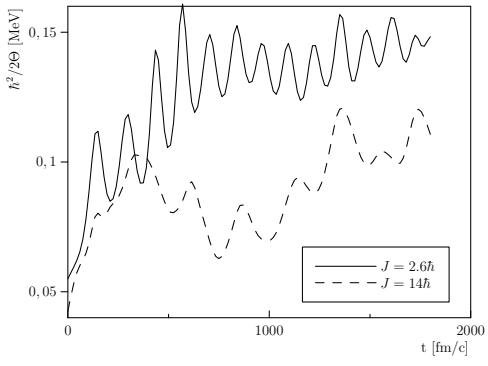

Fig. 11. Coefficient of the rotational energy, $\hbar^{2} / 2 \Theta$ calculated using the TDHF method for two situations: $E=3.3 \mathrm{MeV}$ with $b=8 \mathrm{fm}$, corresponding to $J=2.6 \hbar$, and $E=18 \mathrm{MeV}$ for the same impact parameter with $J=14 \hbar$.

shows the starting conf guration; the initial velocities are in the $x$-direction. Upon contact the two nuclei are strongly attracted and form a compound system that rotates while also undergoing strong vibrations as indicated by the other snapshots.

To illustrate the behavior of the moment of inertia we show its time development for two typical cases in Fig. 11. The moment of inertia is calculated from the instantaneous density as the rigid-body value. They correspond to two different angular momenta at the same impact parameter of $8 \mathrm{fm}$, which is selected to get the system to coalesce into a strongly deformed rotating conf guration; the curves also show the quite large oscillations caused by the shape changes. Yet the tendency appears to follow that of the cranked calculations: for the small angular momentum it goes to much smaller moment of inertia, while for $J=14 \hbar$ the system spends quite a long time oscillating around the moment of inertia found in the cranking calculations before relaxing to a slightly smaller value.

\section{Summary}

In this work we have performed microscopic dynamical calculations of nuclear collisions to study the formation of a metastable linear chain state of ${ }^{12} \mathrm{C}$. The time evolution as calculated using the TDHF equations shows a characteristic quasiperiodic exchange of alpha-like clusters in the density function corresponding to a quasiperiodic motion along a static Hartree-Fock potential energy surface, 


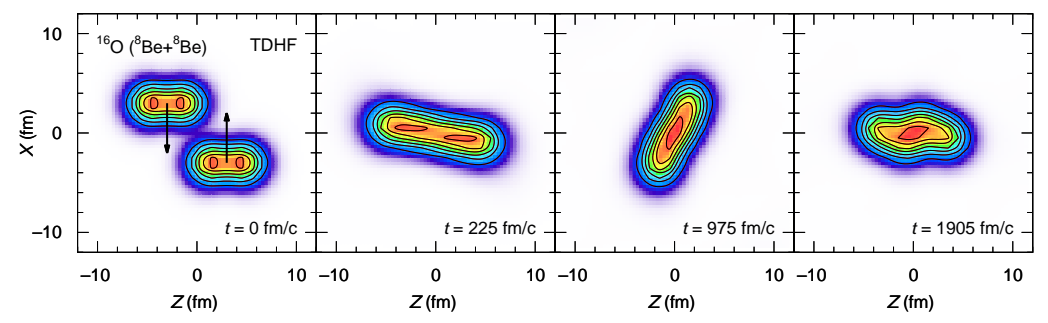

Fig. 10. Snapshots of the total nucleon density calculated using the TDHF method for the ${ }^{8} \mathrm{Be}+{ }^{8} \mathrm{Be}$ collision. The impact parameter is 6 $\mathrm{fm}$ and the relative energy is $2 \mathrm{MeV}$. We normalize the color to the density distribution at the maximum of each plot.

which is studied using the density constraint procedure. We have shown that the calculations lead to the formation of a metastable linear chain state of three alpha-like clusters which subsequently decays to a lower energy triangular alpha-like conf guration before acquiring a more compact shape. This is the frst observation of such mode changes in TDHF calculations and the results seem to be analogous to the suggested astrophysical mechanism for the formation of ${ }^{12} \mathrm{C}$ nuclei.

For the $3 \alpha$-chain conf guration of ${ }^{16} \mathrm{O}$ we found a stabilization due to centrifugal forces for a range of angular momenta not including zero. This is a new situation in cranked mean-f eld calculations. An investigation of the presence of a chain structure in collisions of ${ }^{8} \mathrm{Be}+{ }^{8} \mathrm{Be}$ shows a rotating compound system of roughly the right deformation, but with superimposed strong shape oscillations.

\section{Acknowledgments}

This work has been supported by the U.S. Department of Energy under grant No. DE-FG02-96ER40963 with Vanderbilt University, by the German BMBF under contract No. 06FY9086, and by the GCOE program "The Next Generation of Physics, Spun from Universality and Emergence" from MEXT of Japan. A.S.U. thanks for support by the Hessian LOEWE initiative through the Helmholtz International Center for FAIR; J.A.M thanks the Japan Society for the Promotion of Science (JSPS) for an invitation fellowship for research in Japan (short-term).

\section{References}

1. J. A. Maruhn, M. Kimura, S. Schramm, P.-G. Reinhard, H. Horiuchi and A. Tohsaki-Suzuki, Phys. Rev. C 74, 044311 (2006).

2. N. Itagaki and S. Okabe, Phys. Rev. C 61, 044306 (2000).

3. M. Ito, N. Itagaki, H, Sakurai and K. Ikeda, Phys. Rev. Lett. 100, 182502 (2008).

4. F. Hoyle, Astrophys. J. Suppl. 1, 121 (1954).

5. H. Morinaga, Phys. Rev. 101, 254 (1956).

6. K. Ikeda, N. Takigawa and H. Horiuchi, Prog. Theor. Phys. Suppl. Extra Number 464 (1968).

7. A. Tohsaki, H. Horiuchi, P. Schuck, and G. Röpke, Phys. Rev. Lett. 87, 192501 (2001).
8. P. Chevallier et al., Phys. Rev. 160, 827 (1961).

9. Y. Suzuki, H. Horiuchi and K. Ikeda, Prog. Theor. Phys. 471517 (1972).

10. H. Horiuchi et al., Prog. Theor. Phys. Suppl. 52, 89 (1972).

11. A.S. Umar, J.A. Maruhn, N. Itagaki, and V.E. Oberacker, Phys. Rev. Lett. 104212503 (2010).

12. N. Itagaki et al., Phys. Rev. C 64, 014301 (2001), N. Itagaki, W. von Oertzen, and S. Okabe Phys. Rev. C 74067304 (2006).

13. J.A. Maruhn, N. Loebl, N. Itagaki, and M. Kimura, Nucl. Phys. A 833 (2010).

14. J. W. Negele, Rev. Mod. Phys. 54, 913 (1982).

15. A. S. Umar, M. R. Strayer, R. Y. Cusson, P. -G. Reinhard, and D. A. Bromley, Phys. Rev. C 32, 172 (1985).

16. R. Y. Cusson, P. -G. Reinhard, M. R. Strayer, J. A. Maruhn, and W. Greiner, Z. Phys. A 320, 475 (1985).

17. A. S. Umar and V. E. Oberacker, Phys. Rev. C 74, 021601(R) (2006).

18. A. S. Umar and V. E. Oberacker, Phys. Rev. C 77, 064605 (2008).

19. A.S. Umar and V. E. Oberacker, Eur. Phys. J. A 39, 243 (2009).

20. E. Chabanat, P. Bonche, P. Haensel, J. Meyer and R. Schaeffer, Nucl. Phys. A635, 231 (1998); A643, 441(E) (1998).

21. A. S. Umar and V. E. Oberacker, Phys. Rev. C 73, 054607 (2006).

22. N. Itagaki, W. von Oertzen, and S. Okabe, Phys. Rev. C 74, 067304 (2006).

23. P.-G. Reinhard, and R. Y. Cusson, Nucl. Phys. A378, 418 (1982).

24. E. Chabanat, P. Bonche, P. Haensel, J. Meyer, and R. Schaeffer, Nucl. Phys. A627, 710 (1997).

25. P.-G. Reinhard and H. Flocard, Nucl. Phys. A584, 467 (1995).

26. N. Itagaki, A. Ohnishi, and K. Katō, nucl-th/9606506 